\title{
Concomitant Gallstone Disease Was Associated With a Worse Prognosis in Non-Cirrhotic Patients With Primary Biliary Cholangitis
}

\section{Sha Chen}

Capital Medical University

Mengqi Li

Capital Medical University

Buer Li

Capital Medical University

Tingting LV

Capital Medical University

Shuxiang Li

Capital Medical University

Min Li

Capital Medical University

Yuanyuan Kong

Capital Medical University

Dong Zhang

Capital Medical University

Hong Ma

Capital Medical University

Xiaojuan Ou

Capital Medical University

Hong You

Capital Medical University

Weijia Duan

Capital Medical University

Jidong Jia ( $\nabla$ jia_jd@ccmu.educ.cn )

Capital Medical University https://orcid.org/0000-0002-4673-8890

\section{Research Article}

Keywords: Primary biliary cirrhosis, gallstones, risk factors, prevalence, prognosis, survival, autoimmune liver disease, Chinese, cholestasis, bile acids, intestinal microbiota 
Posted Date: June 24th, 2021

DOl: https://doi.org/10.21203/rs.3.rs-643876/v1

License: (c) (i) This work is licensed under a Creative Commons Attribution 4.0 International License. Read Full License 


\section{Abstract}

Background \& Aims: Primary biliary cholangitis (PBC) is a rare disease manifested as intrahepatic cholestasis, whereas gallstone disease is a common disease. Little is known on the prevalence and impact of gallstone disease (GD) on the clinical course of PBC. Therefore, we investigated the prevalence and the impact of GD on the prognosis of PBC in cirrhotic and non-cirrhotic PBC patients in China.

Methods: We retrospectively enrolled PBC patients and followed them by reviewing of electronic records and structured, standardized telephone interviews. GD was defined as gallstones demonstrated on ultrasonography or history of cholecystectomy for gallstones. The primary endpoints of the present study were liver-related death and/or liver transplantation.

Results: Among 985 PBC patients enrolled in the present study, 258 (26.2\%) had GD, with 157 (22.9\%) in non-cirrhotic patients and 101 (33.8\%) in cirrhotic patients. No difference was observed in the prevalence of GD between women and men ( $p>0.05)$. Compared with PBC patients without GD, those with GD were older, more often coexistent type 2 diabetes, and had a more severe liver disease at baseline. Furthermore, those with GD had lower transplant-free survival and higher incidence of hepatic events in non-cirrhotic PBC patients (both $p<0.05$ ). Multivariate Cox regression showed that concomitant GD, older age, male sex, and higher bilirubin were independent risk factors associated with transplant-free survival in noncirrhotic PBC patients.

Conclusions: Concomitant GD was common and an independent risk factor that associated with a worse prognosis in non-cirrhotic PBC patients.

\section{Introduction}

Primary biliary cholangitis (PBC) is a rare cholestatic disease characterized by inflammation and destruction of interlobular bile ducts[1]. The incidence and prevalence of PBC are in an increasing tendency worldwide[2]. Gallstone disease (GD) is one of the most prevalent and costly disorders of all gastrointestinal diseases, which remains a significant public health issue[3]. Cirrhosis is a welldemonstrated factor favoring gallstone formation, and several studies[4-6] have focused on the relationship between GD and liver disease, mainly including non-alcoholic fatty liver disease and chronic hepatitis $\mathrm{C}$ infection. However, little is known on the prevalence and clinical significance of GD in PBC patients.

Therefore, in the present study, we aimed to investigate the prevalence of GD in cirrhotic and non-cirrhotic PBC patients, and explore the impact of GD on the prognosis of PBC patients in China.

\section{Methods}

\section{Study design and patients}


We retrospectively analyzed all PBC patients diagnosed and treated at the outpatient clinics or the inpatient department of the Liver Research Center, Beijing Friendship Hospital, Capital Medical University from January 1, 2000, to December 31, 2020. This study was approved by the ethics committee of the Beijing Friendship Hospital, Capital Medical University. All patients gave verbal consent that granted by the ethical committee.

Patients will be included if they met the diagnostic criteria of PBC based on international guidance[1]. Patients will be excluded if they (1) coexisted with autoimmune hepatitis, chronic viral hepatitis B or $\mathrm{C}$, or drug-induced liver injury; (2) without a standardized dose of ursodeoxycholic acid (UDCA) treatment; (3) with incomplete laboratory data at baseline; or (4) lost to follow up or followed shorter than 12 months.

\section{Baseline data collection}

We collected the baseline medical information of enrolled patients by reviewing the electronic medical records. They included age, sex, body mass index; complete blood count, international normalized ratio (INR); blood biochemistry: alkaline phosphatase, gamma-glutamyl transpeptidase, aminotransferase, albumin, bilirubin, total bile acid (TBA), fasting plasma glucose, triglycerides, total cholesterol, low-density lipoprotein, and high-density lipoprotein; immunology: immunoglobulin $M$, immunoglobulin $G$, antimitochondrial antibody (AMA) or anti-mitochondrial M2 antibody (AMA-M2) status; HBsAg and anti-HBC status; radiology: abdominal ultrasonography, computed tomography (CT), or magnetic resonance imaging (MRI); upper gastrointestinal endoscopy, and liver histology.

\section{Follow-up of the patients}

Two well-trained investigators followed the enrolled PBC patients through reviewing of medical records and structured, standardized telephone interviews. Follow-up data were obtained including smoking habit, alcohol assumption, the diagnosis time and treatment of GD, the diagnosis time of type 2 diabetes, fatty liver, and hypertension.

The occurrence time of liver-related death, liver transplantation (LT), hepatic events including decompensated cirrhosis (hepatic encephalopathy, ascites, or variceal bleeding), and hepatocellular carcinoma $(\mathrm{HCC})$ were recorded. The primary outcomes of the present study were liver-related death and/or LT.

\section{Definition and Diagnosis criteria}

GD was diagnosed according to sonographic findings of echoes with posterior acoustic shadowing within the gallbladder[7] at baseline or during follow-up, or a history of cholecystectomy for gallstones.

Smoking habit was defined as smoking more than 10000 cigarettes lifetime. Alcohol abuse was defined as on average alcohol assumption $>40 \mathrm{~g}$ per day for men or $>20 \mathrm{~g}$ per day for women last longer than 5 years[8]. Type 2 diabetes and hypertension were diagnosed based on guidelines[9, 10]. Past chronic 
hepatitis B virus infection was considered as positive anti-HBc with negative HBsAg. Cirrhosis and HCC were diagnosed according to the published guidelines[11, 12].

\section{Statistical analysis}

Age and albumin were expressed as means with standard deviations and compared using student $t$-tests since they fitted the normal distribution. Other continuous variables were described as median (interquartile range) and compared using the Mann-Whitney U-test between patients with and without GD. Categorical variables including gender, the status of AMA/AMA-M2, smoking habit, alcohol assumption, cirrhosis, and concomitant diseases were expressed as counts and percentages. The chisquared test was used to compare categorical variables above between patients with and without GD.

Transplant-free survival was calculated by Kaplan-Meier curves and the difference was determined by log-rank test. Univariate and multivariate Cox regression was applied to find the risk parameters associated with prognosis. A two-sided $P$ value $<0.05$ was considered significant. The analyses were conducted by using SPSS statistics version 26 .

\section{Results}

\section{Prevalence of gallstone disease in PBC}

A total of 985 UDCA-treated PBC patients were enrolled with strict inclusion and exclusion criteria (Fig. 1). Overall, 258 (26.2\%) PBC patients had concomitant GD at the end of follow up, including 222 patients with present gallstones and 36 with cholecystectomy for gallstones. The prevalence of GD in noncirrhotic and cirrhotic PBC patients were $22.9 \%$ and $33.8 \%$, respectively. Among 852 patients without GD at the diagnosis of PBC, 125 (14.7\%) of them eventually developed GD during follow up.

The prevalence of GD in PBC patients stratified by cirrhosis and gender was shown in Table 1. No difference was observed in the prevalence of GD between women and men both in cirrhotic and noncirrhotic groups $(p>0.05)$.

\section{Baseline characteristics of PBC patients with and without gallstone disease}

The demographic characteristics, laboratory examinations, lifestyle evaluation, and comorbidities of PBC patients with and without GD were shown in Table 2. Overall, PBC patients with GD were older and more often coexistent type 2 diabetes than patients without GD. Additionally, patients with GD were in a more advanced disease stage than those without, characterized by lower albumin and platelet count (PLT), higher bilirubin, INR, and TBA, as well as a higher proportion of cirrhotic patients. However, the frequency of concomitant fatty liver, hypertension and past hepatitis B virus infection was similar between the two groups.

The impact of concomitant gallstone disease on the prognosis of non-cirrhotic and cirrhotic PBC patients 
After a median follow-up time of 5.3 years (range: 1.0-20.9), a total of 100 (10.2\%) PBC patients reached an endpoint (liver-related deaths: $n=76$; LT: $n=24), 24$ (2.4\%) cases developed HCC. Among 845 patients without decompensated cirrhosis at baseline, 172 (20.4\%) cases developed decompensated cirrhosis.

Kaplan-Meier plots for transplant-free survival and cumulative incidence of hepatic events (HCC and/or decompensated cirrhosis) in non-cirrhotic and cirrhotic PBC patients were shown in Fig. 2. In non-cirrhotic PBC patients, those with GD had significantly lower transplant-free survival and higher incidence of hepatic events than in those without GD (both $p<0.05$ ). However, in cirrhotic PBC patients, the transplantfree survival and incidence of hepatic events between patients with and without GD were similar.

\section{Risk factors associated with prognosis}

Univariate and multivariate Cox analyses were performed to identify risk factors associated with hard endpoints (liver-related death and/or LT). We found that concomitant GD, age, male sex, bilirubin, albumin, gama-glutamyl transpeptidase ( $\gamma-G T)$, and TBA were all significant in univariate analysis. However, only concomitant GD, age, male sex, and bilirubin remain significant in multivariable Cox analysis (Table 3).

\section{Discussion}

To our knowledge, this is the first cohort study reporting the prevalence, characteristics, and clinical significance of GD in PBC patients. We found a high prevalence of GD both in non-cirrhotic and cirrhotic PBC patients regardless of sex. PBC patients with GD were older, more often coexistent type 2 diabetes, and associated with a more severe liver disease at baseline than those without GD. More importantly, Cox regression analysis showed that concomitant GD was an independent risk factor that was associated with worse outcomes in non-cirrhotic PBC patients after adjusting for other important variables.

We found a significant higher prevalence of GD in PBC patients (26.2\%) than that in general population, which was reported as 2.3-6.5\% in Chinese adults[13]. Not surprisingly, a high prevalence of GD (33.6\%) was shown in cirrhotic PBC patients, which could be mainly explained by cirrhosis and a high proportion of females, two well-known risk factors for developing GD[14, 15]. However, we also identified a high prevalence of GD in non-cirrhotic and male PBC patients, indicating that there might be other potential mechanisms for gallstone formation in these particular populations. The following factors may be explanations for the high prevalence of GD in PBC patients.

Firstly, the reduced bile acids pool due to cholestasis broke the balance of bile contents. Normally, cholesterol, lecithin, and cholic acids maintain homeostasis by micelles formation[16]. Under the circumstances of cholestasis, part of the bile acids will be transported into the systemic circulation instead of into the bile canaliculus due to excretion impairment[17], resulting in the reduction of the bile acid pool and cholesterol supersaturation. At the same time, the free bilirubin solubility in bile also 
depends on bile acids concentration, the decrease of the latter can lead to the formation of pigment stones.

Secondly, the altered biliary bile acids composition in PBC patients favored the gallstone formation. Studies have proved that chenodeoxycholic acid (CDCA) can lower the cholesterol saturation in bile and dissolve cholesterol stones by suppressing cholesterol synthesis and secretion[18, 19]. In contrast, cholic acid (CA) can increase the intestinal cholesterol absorption[20]. In a study that enrolled 98 treatmentnaïve $\mathrm{PBC}$ patients and 14 healthy controls, Combes $B$ et al.[21] found an increased $C A$ and decreased CDCA in bile in PBC patients, which means that increased CA to CDCA ratio in PBC patients provided a favorable condition for gallstones formation.

Thirdly, the biliary infection caused by the imbalance of cholestasis-bile acids-intestinal microbiota triangle also played a crucial role. Nowadays, the crosstalk between bile acids and gut microbiota in the pathogenesis of PBC has arisen great interest in investigators. On the one hand, gut microbiota can regulate secondary bile acids metabolism and modulate hepatic bile acid synthesis[22]; on another hand, bile acids can shape gut microbiota by inhibiting the overgrowth of certain bacteria[23]. In PBC, the reduced bile acids secretion, altered gut microbiota[24] leads to bacterial translocation and biliary infection, eventually contributing to the formation of diverse types of gallstones.

Finally, impaired gallbladder mobility caused by constant stimulation of hydrophobic bile acids due to cholestasis might be another factor[25]. Besides, treatment with fibrates might promote cholesterol gallstone formation since PPARa reduces the transcription of CYP7A1, a rate-limiting enzyme regulating bile acids synthesis from cholesterol[26]. Putting together, the high prevalence of GD in PBC patients might be explained by reduced total bile acid pool, altered biliary bile acids composition, imbalance of gut microbiota, impaired gallbladder mobility, and drug treatment like fibrates.

Another interesting finding in the present study was that concomitant GD was independently associated with worse outcomes in non-cirrhotic PBC patients. Since cirrhosis is the most important risk factor associated with prognosis and GD is more prevalent in cirrhotic patients, we analyzed the impact of GD on prognosis stratified by cirrhosis. For non-cirrhotic PBC patients, only four risk factors including age, male sex, bilirubin, together with GD remain significant in the multivariate Cox regression analysis. The significance of the other three parameters was widely reported in many cohorts[27, 28], while none of the studies focused on the impact of GD on the prognosis of PBC. In our cohort, although patients with GD were older and more often coexistent metabolism-related disease at baseline than those without in noncirrhotic PBC patients, the disease severity at baseline was comparable between the two groups (supplementary table 1). Besides, considering that variables that might be associated with prognosis were basically all involved in the univariate Cox analysis and GD remains significant after adjusting for age and other important variables, we believe the results were reliable. For cirrhotic PBC patients, concomitant GD was not significant in univariate Cox analysis.

The mechanism that GD only affected the prognosis of non-cirrhotic patients was unknown. One hypothesis was that concomitant GD in the early stage of the disease means that the patients had a 
more disordered biliary and intestinal microenvironment which results in a worse prognosis. In turn, the physical presence of gallstones can cause the inflammation of the gallbladder wall and impair gallbladder mobility, further aggravating cholestasis[29]. Once patients entered an advanced stage, the bile acids metabolism and intestinal microenvironment were already disturbed due to cirrhosis no matter with or without gallstones. This further suggests that early intervention is more beneficial to the disease. Indeed, the first-line and second-line drugs for the treatment of PBC including UDCA and obeticholic acid (OCA) are also effective drugs for the treatment of gallstones.

Our study suggested that early prevention or treatment of gallstones might improve the prognosis of noncirrhotic PBC patients, providing a novel way of thinking for therapy. Lifestyle modification and management of metabolism-related diseases might be the first choice since there was no other effective medication. Other potential therapy targeting gut-liver axis like gut microbiota or gallbladder mobility might another choice. Multiple works waiting to be done in the future, what are the categories of gallstones in non-cirrhotic and cirrhotic PBC patients? Is GD a risk factor for other cholestatic liver diseases like primary sclerosing cholangitis? The answers remain to be investigated.

There are some limitations in the present study. First, the diagnosis of GD was made by other radiologists in routine abdominal ultrasonography instead of the investigators of the study. However, we believe the results were reliable because of the availability and operability of the ultrasonography. Second, we were not able to directly compare the prevalence between PBC patients and healthy controls since we didn't include sex- and age-matched healthy populations. Third, some of the laboratory data were missing because of the retrospective nature.

In conclusion, a high prevalence of GD was found in PBC patients regardless of sex, concomitant GD was an independent risk factor for a worse prognosis in non-cirrhotic PBC patients.

\section{Declarations}

Funding This work is funded by National Natural Science Foundation of China (82000533) and National Natural Science Foundation of China (81770598).

Conflicts of interest The authors declare that they have no conflict of interest.

Ethics approval All procedures followed were in accordance with the ethical standards of the responsible committee on human experimentation (institutional and national) and with the Helsinki Declaration of 1975, as revised in 2008. The ethics committee of the Beijing Friendship Hospital, Capital Medical University reviewed and approved the study.

Consent to participate All patients gave verbal consent that granted by the ethical committee.

Consent for publication Written informed consent for publication was obtained from all participants.

Availability of data and material Not applicable. 
Code availability Not applicable.

Authors' contributions Professor Jidong Jia and Doctor Weijia Duan designed, revised and finalized the manuscript; Doctor Sha Chen searched the literature, analyzed the data and drafted the manuscript; Doctors Mengqi Li and Buer Li followed patients by reviewing electronic medical records and telephone interviews; Doctors Sha Chen, Mengqi Li, Buer Li, Shuxiang Li, Tingting Lv, and Shan Shan collected the clinical data of all PBC patients; Min Li and professor Yuanyuan Kong provided suggestions for statistical analysis; Professors Dong Zhang, Hong Ma, Xiaojuan Ou, and Hong You critically reviewed and revised the manuscript. All authors read and approved the final manuscript.

\section{References}

1. Lindor KD, Bowlus CL, Boyer J, et al. Primary Biliary Cholangitis: 2018 Practice Guidance from the American Association for the Study of Liver Diseases. Hepatology. 2019;69:394-419.

2. Lv T, Chen S, Li M, et al. Regional variation and temporal trend of primary biliary cholangitis epidemiology: A systematic review and meta-analysis. J Gastroenterol Hepatol. 2020;36:1423-34.

3. Sandler RS, Everhart JE, Donowitz M, et al. The burden of selected digestive diseases in the United States. Gastroenterology. 2002;122:1500-11.

4. Bini EJ, McGready J. Prevalence of gallbladder disease among persons with hepatitis C virus infection in the United States. Hepatology. 2005;41:1029-36.

5. Fracanzani AL, Valenti L, Russello M, et al. Gallstone disease is associated with more severe liver damage in patients with non-alcoholic fatty liver disease. PLoS One. 2012;7:e41183.

6. Yilmaz Y, Ayyildiz T, Akin H, et al. Gallstone disease does not predict liver histology in nonalcoholic fatty liver disease. Gut Liver. 2014;8:313-7.

7. Bortoff GA, Chen MY, Ott DJ, et al. Gallbladder stones: imaging and intervention. Radiographics. 2000;20:751-66.

8. Li YM, Fan JG, National Workshop on Fatty. L, et al. Guidelines of prevention and treatment for alcoholic liver disease (2018, China). J Dig Dis. 2019;20:174-80.

9. American Diabetes A. 2. Classification and Diagnosis of Diabetes: Standards of Medical Care in Diabetes-2019. Diabetes Care. 2019;42:13-28.

10. Williams B, Mancia G, Spiering W, et al. 2018 ESC/ESH Guidelines for the management of arterial hypertension: The Task Force for the management of arterial hypertension of the European Society of Cardiology and the European Society of Hypertension: The Task Force for the management of arterial hypertension of the European Society of Cardiology and the European Society of Hypertension. J Hypertens. 2018;36:1953-2041.

11. Suk KT, Baik SK, Yoon JH, et al. Revision and update on clinical practice guideline for liver cirrhosis. Korean J Hepatol. 2012;18:1-21.

12. Bruix J, Sherman M, Llovet JM, et al. Clinical management of hepatocellular carcinoma. Conclusions of the Barcelona-2000 EASL conference. European Association for the Study of the Liver. J Hepatol 
2001;35:421-430.

13. Editorial Board of Chinese Journal of Digestion. Consensus on diagnosis and treatment of chronic cholecystitis and gallstones in China (2018). J Clin Hepatol. 2019;35:1231-6. (in Chinese).

14. Conte D, Fraquelli M, Giunta M, et al. Gallstones and liver disease: an overview. J Gastrointestin Liver Dis. 2011;20:9-11.

15. Shaffer EA. Epidemiology and risk factors for gallstone disease: has the paradigm changed in the 21st century? Curr Gastroenterol Rep. 2005;7:132-40.

16. Itani M, Dubinsky TJ. Physical Chemistry of Bile: Detailed Pathogenesis of Cholelithiasis. Ultrasound Q. 2017;33:229-36.

17. Li Y, Tang R, Leung PSC, et al. Bile acids and intestinal microbiota in autoimmune cholestatic liver diseases. Autoimmun Rev. 2017;16:885-96.

18. Adler RD, Bennion LJ, Duane WC, et al. Effects of low dose chenodeoxycholic acid feeding on biliary lipid metabolism. Gastroenterology. 1975;68:326-34.

19. Einarsson K, Grundy SM. Effects of feeding cholic acid and chenodeoxycholic acid on cholesterol absorption and hepatic secretion of biliary lipids in man. J Lipid Res. 1980;21:23-34.

20. Murphy C, Parini P, Wang J, et al. Cholic acid as key regulator of cholesterol synthesis, intestinal absorption and hepatic storage in mice. Biochim Biophys Acta. 2005;1735:167-75.

21. Combes B, Carithers RL Jr, Maddrey WC, et al. Biliary bile acids in primary biliary cirrhosis: effect of ursodeoxycholic acid. Hepatology. 1999;29:1649-54.

22. Liu HX, Keane R, Sheng L, et al. Implications of microbiota and bile acid in liver injury and regeneration. J Hepatol. 2015;63:1502-10.

23. Watanabe M, Fukiya S, Yokota A. Comprehensive evaluation of the bactericidal activities of free bile acids in the large intestine of humans and rodents. J Lipid Res. 2017;58:1143-52.

24. Tang $\mathrm{R}$, Wei $\mathrm{Y}$, Li Y, et al. Gut microbial profile is altered in primary biliary cholangitis and partially restored after UDCA therapy. Gut. 2018;67:534-41.

25. Xiao ZL, Rho AK, Biancani P, et al. Effects of bile acids on the muscle functions of guinea pig gallbladder. Am J Physiol Gastrointest Liver Physiol. 2002;283:G87-94.

26. Lammert F, Gurusamy K, Ko CW, et al. Gallstones Nat Rev Dis Primers. 2016;2:16024.

27. Chen $\mathrm{S}$, Duan $\mathrm{W}$, You $\mathrm{H}$, et al. A brief review on prognostic models of primary biliary cholangitis. Hepatol Int. 2017;11:412-8.

28. Myers RP, Shaheen AA, Fong A, et al. Epidemiology and natural history of primary biliary cirrhosis in a Canadian health region: a population-based study. Hepatology. 2009;50:1884-92.

29. Domeyer PJ, Sergentanis TN, Zagouri F, et al. Chronic cholecystitis in elderly patients. Correlation of the severity of inflammation with the number and size of the stones. In Vivo. 2008;22:269-72.

\section{Tables}


Table 1. Prevalence of gallstone disease in PBC patients stratified by cirrhosis and gender

\begin{tabular}{|lllll|}
\hline & Overall & Women $(\mathrm{n}=855)$ & Men $(\mathrm{n}=130)$ & $P$ \\
\hline Cirrhotic $(\mathrm{n}=299)$ & $33.8 \%$ & $34.7 \%$ & $29.2 \%$ & 0.509 \\
\hline Non-cirrhotic $(\mathrm{n}=686)$ & $22.9 \%$ & $21.9 \%$ & $30.5 \%$ & 0.092 \\
\hline
\end{tabular}

$P$ values refer to comparisons of prevalence between women and men.

Table 2. Baseline characteristics of PBC patients with and without gallstone disease 


\begin{tabular}{|c|c|c|c|}
\hline Characteristics & $\begin{array}{l}\text { Patients } \\
\text { with GD ( } n=258)\end{array}$ & $\begin{array}{l}\text { Patients } \\
\text { without GD (n=727) }\end{array}$ & $P$ value \\
\hline Age at diagnosis, years & $56.5 \pm 11.5$ & $53.0 \pm 11.5$ & $<0.001$ \\
\hline Females, n (\%) & 219 (84.9) & $636(87.5)$ & 0.286 \\
\hline AMA/AMA-M $2(+)$, n (\%) & $230(89.1)$ & $631(86.8)$ & 0.382 \\
\hline ALP, U/L & $223(148-394)$ & $226(143-398)$ & 0.911 \\
\hline $\mathrm{Y}-\mathrm{GT}, \mathrm{U} / \mathrm{L}$ & $231(125-420)$ & $241(110-463)$ & 0.776 \\
\hline ALT, U/L & $61(35-99)$ & $63(37-107)$ & 0.539 \\
\hline AST, U/L & $64(43-105)$ & $64(42-105)$ & 0.688 \\
\hline ALB, g/L & $37.3 \pm 6.1$ & $38.8 \pm 5.4$ & 0.001 \\
\hline TBIL, $\mu \mathrm{mol} / \mathrm{L}$ & $19.5(13.1-33.7)$ & $16.1(11.9-26.8)$ & 0.001 \\
\hline PLT, 10^9/L & $151(102-210)$ & $184(118-239)$ & 0.012 \\
\hline INR & $1.04(0.96-1.14)$ & $1.01(0.95-1.08)$ & 0.021 \\
\hline $\operatorname{lgG}, \mathrm{mg} / \mathrm{dl}^{\mathrm{a}}$ & $1785(1400-2188)$ & 1651 (1385-2090) & 0.119 \\
\hline $\operatorname{lgM}, \mathrm{mg} / \mathrm{dl}^{\mathrm{b}}$ & $370(232-553)$ & $325(221-505)$ & 0.066 \\
\hline $\mathrm{TBA}, \mu \mathrm{mol} / \mathrm{L}^{\mathrm{c}}$ & $16.3(7.1-34.7)$ & $11.4(5.7-25.1)$ & 0.019 \\
\hline GLU, mmol/L & $5.3(4.8-6.1)$ & $5.1(4.7-5.7)$ & 0.011 \\
\hline $\mathrm{CHE}, \mathrm{KU} / \mathrm{L}^{\mathrm{d}}$ & $6.1(4.4-8.2)$ & $7.1(5.3-8.6)$ & 0.005 \\
\hline $\mathrm{CHOL}, \mathrm{mmol} / \mathrm{L}^{\mathrm{e}}$ & $5.0(3.9-6.2)$ & $5.3(4.4-6.4)$ & 0.159 \\
\hline $\mathrm{TG}, \mathrm{mmol} / \mathrm{L}^{\mathrm{e}}$ & $1.3(0.9-1.9)$ & $1.2(0.9-1.7)$ & 0.297 \\
\hline $\mathrm{LDL}, \mathrm{mmol} / \mathrm{L}^{\mathrm{e}}$ & $2.8(2.2-3.7)$ & $2.9(2.3-3.6)$ & 0.377 \\
\hline $\mathrm{HDL}, \mathrm{mmol} / \mathrm{L}^{\mathrm{e}}$ & $1.3(1.0-1.6)$ & $1.5(1.2-1.8)$ & 0.004 \\
\hline $\mathrm{UA}, \mu \mathrm{mol} / \mathrm{L}^{\mathrm{e}}$ & $280(221-358)$ & 268 (218-323) & 0.089 \\
\hline BMI & $23.0(20.4-25.1)$ & $22.8(20.8-24.8)$ & 0.788 \\
\hline Smoking habit, n (\%) & $27(10.5)$ & $48(6.6)$ & 0.055 \\
\hline Alcohol abuse, n (\%) & $12(4.7)$ & $33(4.5)$ & 1.000 \\
\hline Cirrhosis, n (\%) & $100(38.8)$ & $198(27.2)$ & 0.001 \\
\hline Histological stage I-II, n (\%) ${ }^{f}$ & $43(68.3)$ & $138(73.0)$ & 0.518 \\
\hline
\end{tabular}


Past HBV infection (\%) ${ }^{\mathrm{g}}$

Type 2 diabetes, $\mathrm{n}(\%)$

Concomitant fatty liver, $n(\%)$ $\mathrm{h}$

Hypertension, n (\%)

$65(25.2)$
$145(45.3)$

0.758

$63(47.0)$

$79(10.9)$

0.002

$54(7.4)$

0.285

GD, gallstone disease; AMA, anti-mitochondrial antibody; $A M A-M_{2}$, anti-mitochondrial M2 antibody; ALP, alkaline phosphatase; $\gamma$-GT, gamma-glutamyl transpeptidase; ALT, alanine aminotransferase; AST, aspartate aminotransferase; ALB, albumin; TBIL, total bilirubin; PLT, platelet count; INR, international normalized ratio; IgG, immunoglobulin $\mathrm{G}$; IgM, immunoglobulin $\mathrm{M}$; TBA, total bile acid; GLU, fasting plasma glucose; $\mathrm{CHE}$, cholinesterase; $\mathrm{CHOL}$, total cholesterol; TG, triglycerides; $\mathrm{LDL}$, low-density lipoprotein; HDL, high-density lipoprotein; UA, uric acid; BMI, body mass index.

${ }^{a}$ available in 693 patients; ${ }^{b}$ available in 687 patients; ${ }^{c}$ available in 533 patients; ${ }^{d}$ available in 625 patients; ${ }^{\mathrm{e}}$ available in 484 patients; ${ }^{\mathrm{f}}$ liver biopsy was performed in $252 \mathrm{PBC}$ patients; ${ }^{\mathrm{g}}$ available in 454 patients; ${ }^{\text {h }}$ fatty liver was detected by routine abdominal ultrasonography.

$P$ values refer to comparisons between patients with and without gallstone diseases.

Table 3. Univariate and multivariable Cox regression analysis for transplant-free survival in non-cirrhotic PBC patients 
Univariate analyses

$\mathrm{HR} \quad 95 \% \mathrm{Cl} \quad P$ value $\mathrm{HR} \quad 95 \% \mathrm{Cl} \quad P$ value

\begin{tabular}{lllllll}
\hline Gallstone disease & 2.668 & $1.107-6.430$ & 0.029 & 2.686 & $1.036-6.962$ & 0.042 \\
\hline Age & 1.068 & $1.025-1.112$ & 0.002 & 1.117 & $1.072-1.028$ & 0.001 \\
\hline Male sex & 4.239 & $1.690-10.634$ & 0.002 & 4.385 & $1.666-11.543$ & 0.003 \\
\hline TBIL & 1.019 & $1.012-1.027$ & $<0.001$ & 1.028 & $1.018-1.038$ & $<0.001$ \\
\hline ALB & 0.883 & $0.787-0.990$ & 0.034 & - & - & - \\
\hline Y-GT & 1.001 & $1.000-1.002$ & 0.004 & - & - & - \\
\hline TBA & 1.020 & $1.011-1.030$ & $<0.001$ & - & - & - \\
\hline ALT & 1.001 & $0.998-1.004$ & 0.483 & - & - & - \\
\hline AST & 1.002 & $0.996-1.007$ & 0.556 & - & - & - \\
\hline ALP & 1.001 & $1.000-1.002$ & 0.131 & - & - & - \\
\hline PLT & 0.995 & $0.988-1.002$ & 0.134 & - & - & - \\
\hline IgG & 1.000 & $0.999-1.001$ & 0.485 & - & - & - \\
\hline IgM & 1.001 & $0.999-1.002$ & 0.283 & - & - & - \\
\hline INR & 0.974 & $0.769-1.233$ & 0.827 & - & - & - \\
\hline UA & 0.998 & $0.991-1.004$ & 0.494 & - & - & - \\
\hline BMI & 1.010 & $0.871-1.172$ & 0.896 & - & - & - \\
\hline Hypertension & 1.263 & $0.485-3.289$ & 0.632 & - & - & - \\
\hline Concomitant fatty liver & 0.604 & $0.081-4.520$ & 0.624 & - & - & - \\
\hline Type 2 diabetes & 0.681 & $0.158-2.944$ & 0.607 & - & - & - \\
\hline
\end{tabular}

$\mathrm{Cl}$, confidence interval; TBIL, total bilirubin; ALB, albumin; y-GT, gamma-glutamyl transpeptidase; TBA, total bile acid; ALT, alanine aminotransferase; AST, aspartate aminotransferase; ALP, alkaline phosphatase; PLT, platelet count; IgG, immunoglobulin G; IgM, immunoglobulin M; INR, international normalized ratio; UA, uric acid; BMI, body mass index.

\section{Figures}




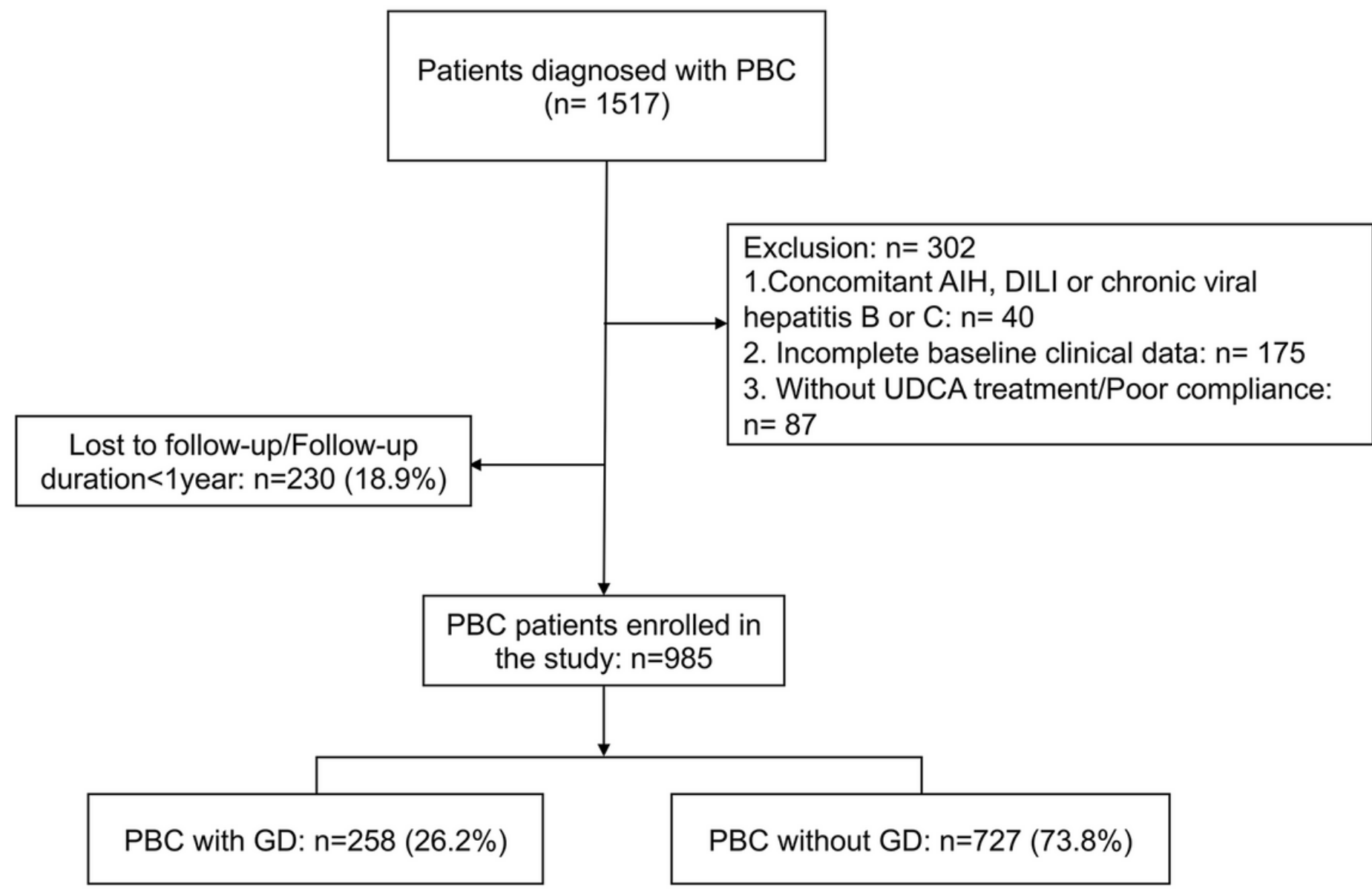

Figure 1

Flowchart of patients selection PBC, primary biliary cholangitis; AlH, autoimmune hepatitis; DILI, druginduced liver injury; UDCA, ursodeoxycholic acid; GD, gallstone disease. 
a

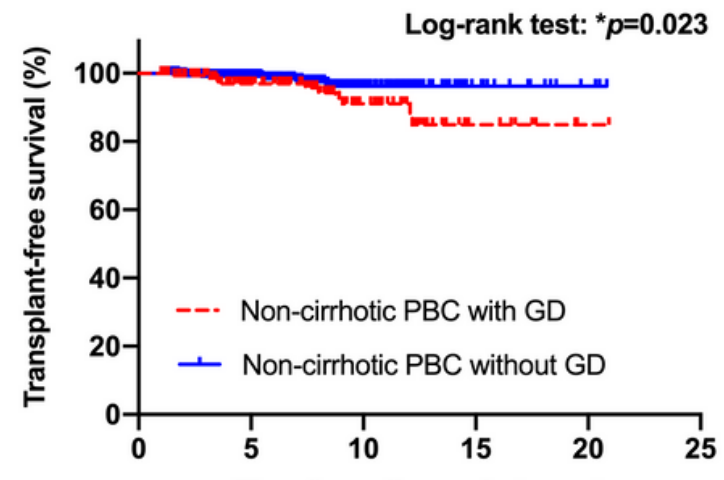

Patients at risk With GD Without GD

Time from diagnosis (years)

$\begin{array}{llllll}157 & 106 & 43 & 10 & 1 & 0 \\ 529 & 297 & 97 & 15 & 4 & 0\end{array}$

Log-rank test: $p=0.687$

C

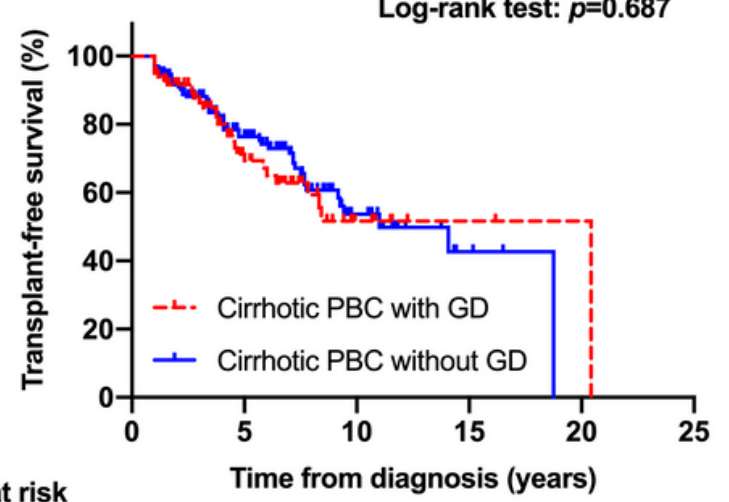

Patients at risk

With GD

Without GD

Time from diagnosis (years)

$\begin{array}{lcccc}35 & 8 & 2 & 1 & 0 \\ 73 & 18 & 4 & 0 & 0\end{array}$
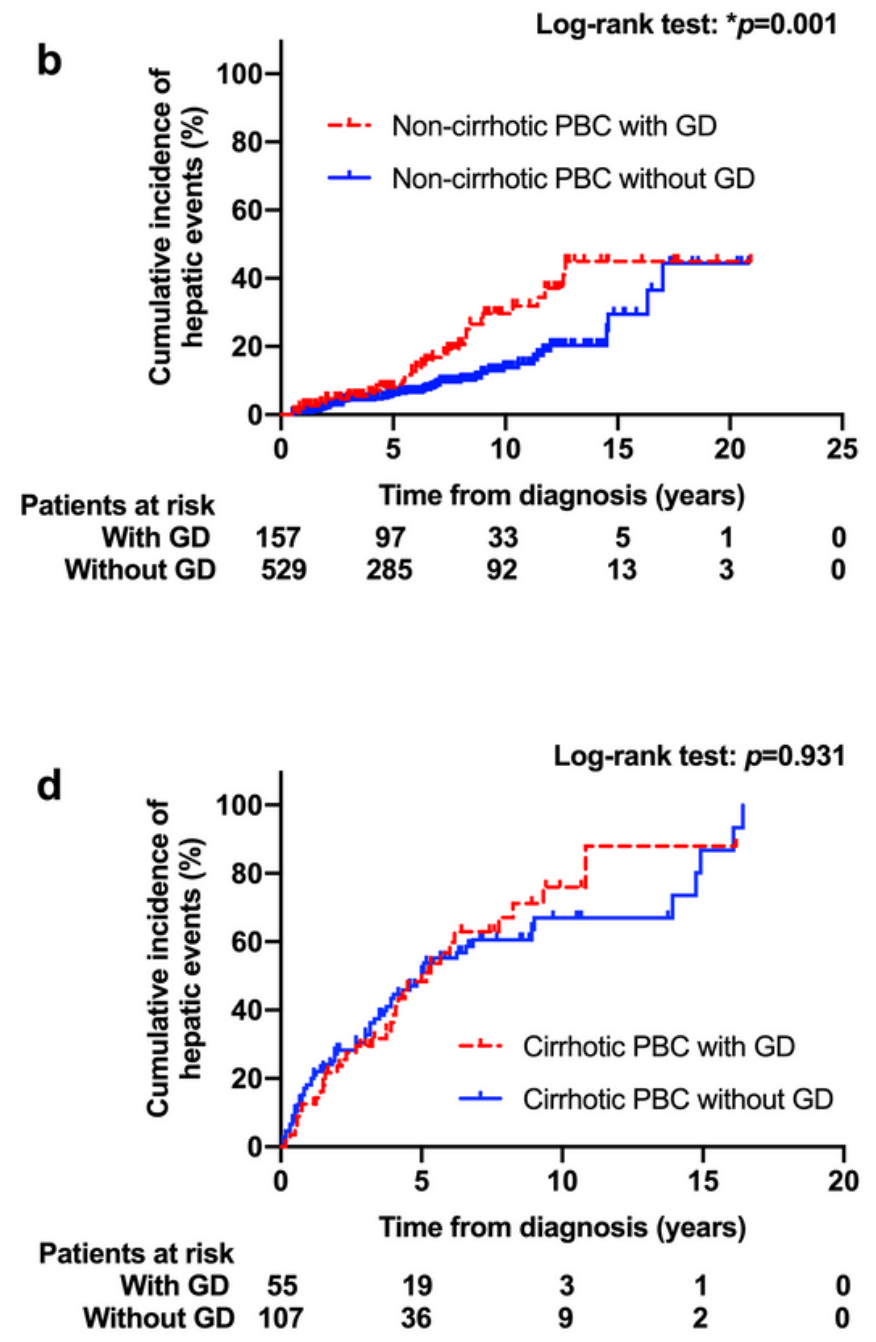

\section{Figure 2}

Kaplan-Meier plots for transplant-free survival and cumulative incidence of hepatic events (decompensated cirrhosis and/or HCC) in PBC patients stratified by with or without GD. Significant lower transplant-free survival and higher incidence of hepatic events were observed in non-cirrhotic PBC patients with GD than in that without GD (both log-rank p<0.05, a, b) HCC, hepatocellular carcinoma; GD, gallstone disease.

\section{Supplementary Files}

This is a list of supplementary files associated with this preprint. Click to download.

- Supplementarytable1.docx 